\title{
Development of a High Precision Infrasonic Sound Sensor
}

\author{
Shi Shuheng ${ }^{1}$ and Wang Wenfan ${ }^{2}$ \\ ${ }^{1}$ Machinery institute, North China University of Water Resources and Electric \\ Power, Zhengzhou, China, 450011, \\ ${ }^{2}$ School of Information Engineering, Shengda Economics Trade \& Management \\ College of Zhengzhou, China, 451191 \\ shuhengshi@163.com
}

\begin{abstract}
Infrasonic wave is the usually sound wave caused by vibrating body at frequency below $20 \mathrm{~Hz}$, which is commonly found in a variety of industry, transportation, nature and living environment. This paper designs a high precision infrasonic sound sensor based on $P V D F$, which is a new piezoelectric material of macromolecule with piezoelectric property. The research results proved that when a given frequency values between $3 \mathrm{~Hz}$ to $30 \mathrm{~Hz}$, the proposed sensor has good performance and high precision, which could meet the design requirements, and has a certain practical value.
\end{abstract}

Keywords: PVDF, infrasonic wave sensor, signal process circuit

\section{Introduction}

Generally speaking, in the acoustic spectrum division, if the frequency of sound is lower than $20 \mathrm{~Hz}$, we call it infrasonic sound, which is made by vibrating and we human could not hear them because the frequency is beyond the limit of audibility $[1,2]$. Infrasonic wave is real exiting in various natural, industrial, transportation and living environment. Such as bridge shaking, cars speed up, and even home stereo system. The infrasonic sound has characteristics of low frequency and long wavelength, these performances make it could spread far, with strong penetrating power and good antijamming. Therefore, the research on the infrasonic wave has become a hot topic recently in many countries, to design infrasound weapons, sound exploration [3, 4], natural disaster prediction [5, 6], medical [7] and so on. The initial research on infrasonic sound is upon weapons, primarily for military surveillance and weapons.

Early to 1990s, U.S. has developed military high-power micro infrasound weapons, and then put it into the simulated field test. In paper [8], the researchers proposed realtime infrasound data processing system based on FGPA (Field Programmable Gata Array), and the use discrete wavelet transforms (DWT) to build a high -pass and a lowpass filter.

Ham presents a classification system with performance results and associated confidence intervals (CIs) to sort four infrasound waves by using parallel RBF neural network [9].

In order to make best use of infrasonic wave signal in the application of forecast earthquake, paper [10] presents the software based on MATLAB software to analyze abnormal infrasound signal, which is very important to forecast earthquake. Shan, et al. presented an infrasound wave system to monitor the process of low carbon steel damage, which confirmed that during the damage phase, it will generate about $2 \mathrm{~Hz}$ infrasound wave signal, by this method, the workers could find out the unqualified steel quickly [11].

Due to the wide range application of infrasonic wave, it is necessary to design an infrasonic sound sensor, which should have very high precision. However, the existing sound sensor to detect sound have some shortcomings, such as sensitivity to low 
frequency range or big volume, which is not convenient to install and require higher environment. Therefore, in this paper, we proposed a smart and portable infrasonic sound sensor with high precision based on PVDF sensors. The rest of this paper is organized as follows. In Section 2, the methodology of design infrasonic sound sensor is introduced, which include the piezoelectric equation and mechanical equation. Section 3 presents the detail whole design for hardware circuit, which is consists of signal extraction module, normalized amplifying circuit, network filter and overload detection module. The results and analysis are given in Section 4. Finally, Section 5 concludes the paper.

\section{Methodology}

\subsection{Piezoeletric Equation}

Piezoelectric equation refers to the relationship between the mechanics upon the piezoelectric materials (such as stress tensor $\mathrm{T}$, deformation tensor $\mathrm{S}$ ) and electrical signal (such as electric field intensity E, electric displacement D). Currently, the most widely used is the first kind of piezoelectric equation, which is as shown in formula (1) and (2).

$$
\begin{gathered}
S=s^{E} T+d^{T} E \\
D=d T+\varepsilon^{T} E
\end{gathered}
$$

Where, $d=[d i j], i=1,2,3 ; j=1,2, \cdots 6$, which is piezoelectric constant matrix, $\varepsilon^{T}$ is dielectric constant matrix when the stress remains unchanged, $\varepsilon^{E}$ is flexible constant matrix when the electric field remains unchanged.

According to the Gauss theorem, we can obtain the equation of electric quantity of any free point on the surface of the electric field, the formula is as shown inError! Reference source not found., and furthermore, the formula could be simplified as (4) if the size of PVDF is small.

$$
\begin{gathered}
Q(t)=Q\left(t_{0}\right)+E_{P}+d_{31} \int_{0}^{1} \int_{0}^{\omega} S d_{x} d_{y} \\
Q(t)=Q\left(t_{0}\right)+E_{P} d_{31} S_{1} l \omega
\end{gathered}
$$

Where, $Q(t)$ and $Q\left(t_{0}\right)$ are the surface electric quantity on the PVDF surface at $t$ and $t_{0}$, respectively, $\mathrm{S}$ is the strain capacity along the drawing direction.

\subsection{Mechanical Model}

The elastic surface equation is as shown in formula(5), under the action of concentrated force $\mathrm{F}$ on the scope of the small deflection.

$$
\varepsilon_{r}=\frac{1}{E}\left(\sigma_{r}-\mu \sigma_{t}\right)=\frac{1}{E} \frac{3 F}{2 \pi h^{2}}\left(1-\mu^{2}\right)\left(\ln \frac{R}{r}-1\right)
$$

Where,

$\mu$ is the Poisson's ratio of material

$\omega$ is the deflection of the diaphragm

$F$ is the force on the diaphragm

$h$ is the thickness of the diaphragm

$E$ is the material elastic modulus

$r$ is the distance to the diaphragm center

$R$ is the radius of diaphragm

We could get the maximum deflection of the diaphragm when $r=0$, and the simplified equation is as shown inError! Reference source not found.. 


$$
\omega_{0}=\frac{3\left(1-\mu^{2}\right)}{4 \pi} \frac{F R^{2}}{E h^{3}}
$$

When $\mathrm{r}=\mathrm{R}$, the simplified equation is as shown in(7).

$$
\omega_{r=R}=-\frac{3 F}{2 \pi h^{2}}
$$

According to the distribution of concentration on the small area, we could get the maximum stress, the corresponding strain formulas are as follows:

The radial stress and strain is as shown in equation(8).

$$
\omega_{r}=\frac{3\left(1-\mu^{2}\right)}{4 \pi} \frac{1}{E h^{3}}\left(R^{2}-r^{2}+2 r^{2} \ln \frac{r}{R}\right) F
$$

The tangential stress and strain is as shown in equation

$$
\varepsilon_{t}=\frac{1}{E}\left(\sigma_{t}-\mu \sigma_{r}\right)
$$

\section{Signal Processing}

\subsection{Overall Design}

Figure1 shows the whole design of signal process circuit, it is consists of three main parts, first is signal extraction part, which extracts weak electric signals from PVDF sensor, and then the second part is normalized amplifying circuit, which is in charge of amplifying the signal to a definite range, after that, the signal will be sent to a network filter, which is made up of high-pass filter circuit and low-pass filter circuit. Furthermore, we design an overload detection module to monitor system ensure it is in normal condition. The details of these modules will be introduced below.

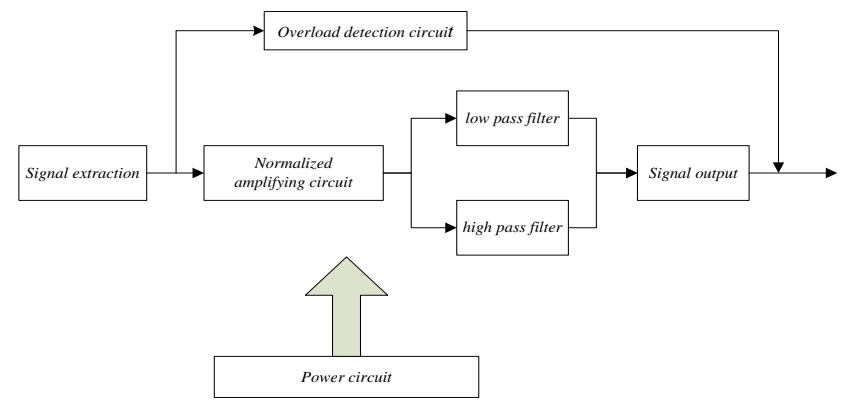

Figure 1. Overall Design of Signal Process Circuit

\subsection{Normalized Amplifying Circuit}

Due to the sensitivity of the sensor is not the same, if we use different sensors, the output voltage generated by the amplifying circuit is also different. In order to control the output voltage within a certain range, it is essential to design a normalization amplifying circuit, which is as shown in Figure2. R12 is a digital potentiometer, which is controlled by the microcontroller, besides that, R14 is a trimming potentiometer. The gain $\mathrm{K}$ of normalized amplifying circuit is shown as in formula(10), it is easily seen that, if we adjust the R12 and R14 to the appropriate value, and we will be able to get a match with the designed sensor gain value.

$$
K=\frac{U_{O 3}}{U_{O 2}}=-\frac{R_{13}+R_{14}}{R_{12}+R_{11}}
$$




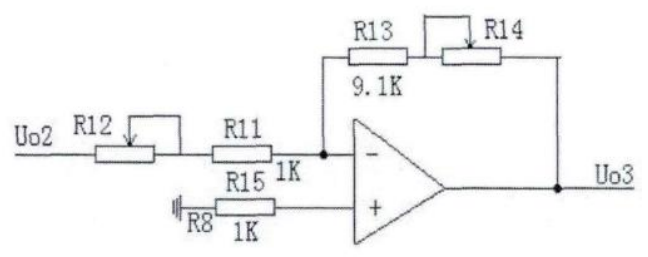

Figure 2. Schematic Diagram Normalized Amplifying Circuit

\subsection{Filter Circuit}

The filter circuit is one of the most important modules to get the precision of the measurement. Hence, the design of the filter circuit is very important. The filter circuit is consists of two parts, one is he high-pass filter circuit, and the other is low-pass circuit. Low-pass filter circuit in the system will only make the signal pass, whose frequency is lower than cut-off frequency, and filter out the signal, whose frequency is higher than cutoff frequency. On the other hand, the function of high-pass module is on the contrary. The combination of low-pass circuit and high-pass-circuit could control select the signal we want to reserve.

The circuit of low-pass module is designed by MAX293, which is a programmable low pass filter. First, we will use microcontroller to measure the frequency of input signal and then, by controlling the analog switch to achieve frequency filtering. The circuit of highpass module is shown as in Figure3, where C24, R15, U5 constitute a first-order RC highpass filter, which will filter the DC component in the input signal, the cut-off frequency of high-pass module is calculated by equation(11) .

$$
f_{h}=\frac{1}{2 \pi R_{15} C_{24}}
$$

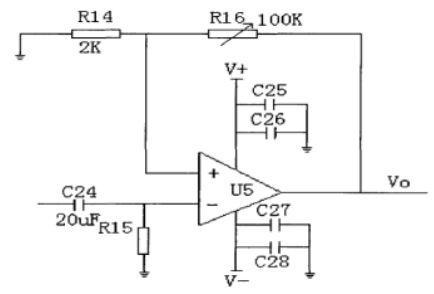

Figure 3. Schematic Diagram of High-pass Filter Circuit

Furthermore, at the same time, this circuit is also could amplify the input signal, which is controlled by the proportion amplifier circuit consisted by the resistance R14 and R16.

\subsection{Overload Detection Circuit}

In order to ensure that the output signal is not distortion, we designed the overload monitoring module and the circuit is as shown in Figure4. The operating principle of overload detection circuit is to monitor the amplifier by microcontroller, when the output signal is beyond a certain range, overload indicator light will be lighted, which implies the circuit is overload.

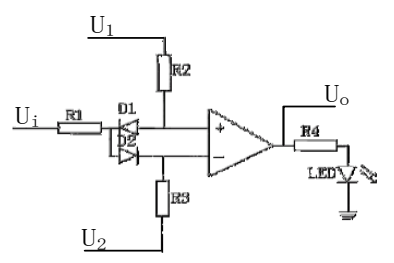

Figure 4.Schematic Diagram of Overload Detection Circuit 
Figure 5 shows the specific working process of overload detection circuit is as follows:

(1) Ui is greater than U1, D1 is cut-off, and D2 is turn-on, the output signal, Uo is high level;

(2) Ui is lower than U2, D1 is turn-on, and D2 is cut-off,, the output signal, Uo is high level;

(3) Ui is between U1 and U2, both D1 and D2 are turn-on, and the output signal, Uo is low level;

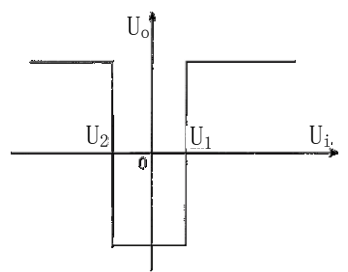

Figure 5. Output Waveform of Detection Circuit

\section{Results and Discussions}

The aim of this part is to verify the performance of infrasonic sound sensor designed in this paper, and the experiment could be divided into two parts. The first part is generating infrasonic sound, which is done by signal generator, and the signal is $1 \sim 20 \mathrm{~Hz}$ sine wave. On the other hand, the other part is receiving the infrasonic sound, which is done by proposed system in this paper.

In order to make the measurement results more convincing, we extend the frequency of generating signal from $1 \sim 20 \mathrm{~Hz}$ to $1 \sim 34 \mathrm{~Hz}$, and besides that, we take the average method to get more precision data, the calculation formulas of frequency and voltage of signal is as shown in the equation (12) and (13), respectively.

$$
\begin{aligned}
& \bar{f}=\frac{1}{n} \sum_{1}^{n} f_{i} \\
& \bar{U}=\frac{1}{n} \sum_{1}^{n} U_{i}
\end{aligned}
$$

The measurement results are as shown in Table1. In order to analyze the results, Figure6 are presented, which shows the change curve of output frequency of infrasonic sound sensor along with real frequency of signal generated by signal generator; and Figure7 shows the output voltage of proposed system along with real frequency of signal generated by generator.

Table 1. Absolute Error of Predicting

\begin{tabular}{|l|l|l|l|}
\hline No. & $\mathrm{O}-\mathrm{F} / \mathrm{Hz}$ & $\mathrm{M}-\mathrm{F} / \mathrm{Hz}$ & $\mathrm{M}-\mathrm{V} / \mathrm{mV}$ \\
\hline 1 & 1 & 0.2 & 0.2 \\
\hline 2 & 2 & 0.5 & 0.2 \\
\hline 3 & 3 & 3.32 & 12.12 \\
\hline 4 & 4 & 4.14 & 12.21 \\
\hline 5 & 5 & 5.43 & 12.3 \\
\hline 6 & 6 & 6.61 & 12.55 \\
\hline 7 & 7 & 7.58 & 13.01 \\
\hline 8 & 8 & 8.56 & 13.22 \\
\hline 9 & 9 & 9.37 & 13.53 \\
\hline 10 & 10 & 10.22 & 13.74 \\
\hline 11 & 11 & 11.29 & 13.98 \\
\hline
\end{tabular}




\begin{tabular}{|c|c|c|c|}
\hline 12 & 12 & 11.35 & 14.11 \\
\hline 13 & 13 & 12.51 & 14.42 \\
\hline 14 & 14 & 13.78 & 14.65 \\
\hline 15 & 15 & 14.25 & 15.97 \\
\hline 16 & 16 & 15.49 & 15.25 \\
\hline 17 & 17 & 16.23 & 15.73 \\
\hline 18 & 18 & 17.76 & 15.1 \\
\hline 19 & 19 & 18.68 & 15.26 \\
\hline 20 & 20 & 20.43 & 15.4 \\
\hline 21 & 21 & 21.3 & 15.61 \\
\hline 22 & 22 & 22.7 & 15.88 \\
\hline 23 & 23 & 23.6 & 16 \\
\hline 24 & 24 & 24.8 & 16.21 \\
\hline 25 & 25 & 25.9 & 16.45 \\
\hline 26 & 26 & 26.5 & 16.7 \\
\hline 27 & 27 & 27.6 & 17 \\
\hline 28 & 28 & 28.4 & 17.27 \\
\hline 29 & 29 & 29.3 & 17.51 \\
\hline 30 & 30 & 30.5 & 17.77 \\
\hline 31 & 31 & 8 & 7.33 \\
\hline 32 & 32 & 2 & 5 \\
\hline 33 & 33 & 0 & 0 \\
\hline 34 & 34 & 0 & 0 \\
\hline
\end{tabular}

From Figure6, we can see that, first when the frequency is below $3 \mathrm{~Hz}$, the output frequency of sensor is clearly not right, with the output results is $0.2 \mathrm{~Hz}$ according to $1 \mathrm{~Hz}$, and $0.5 \mathrm{~Hz}$ according to $2 \mathrm{~Hz}$. This implies the lower limit frequency of this proposed infrasonic sound sensor is $3 \mathrm{~Hz}$. Secondly, when the input frequency is higher than $30 \mathrm{~Hz}$, the output frequency decrease sharply and down to zero, the reasons for this phenomenon are consists of two parts, one is the higher limit frequency of sensor is about $30 \mathrm{~Hz}$, and the other reason is that the cut-off frequency of low-pass filter circuit is designed at $30 \mathrm{~Hz}$. Thirdly, when the frequency is between $3 \mathrm{~Hz}$ and $30 \mathrm{~Hz}$, the output results show perfect linearity, which suggests that the designed sensor is success.

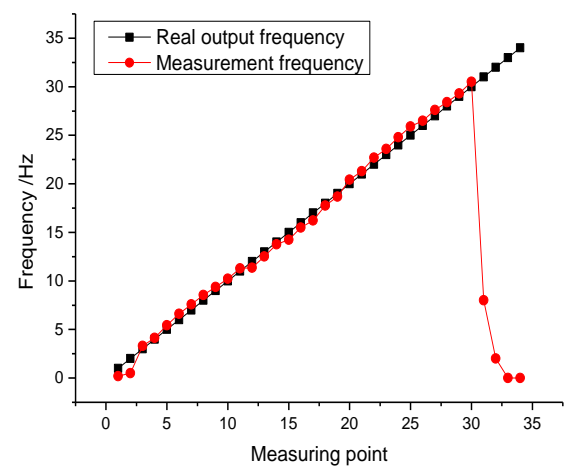

Figure 6. Measurement of Output Frequency

The same conclusion could be got through Figure 7, when the frequency is lower than $3 \mathrm{~Hz}$ of higher than $30 \mathrm{~Hz}$; the output voltage is almost zero. Otherwise, the change curve of voltage is slowly rising with frequency. It is could be drawn a conclusion that:

(1) The low limit frequency of infrasonic sound sensor proposed in this paper is $3 \mathrm{~Hz}$. 
(2) The high limit frequency of infrasonic sound sensor proposed in this paper is $30 \mathrm{~Hz}$.

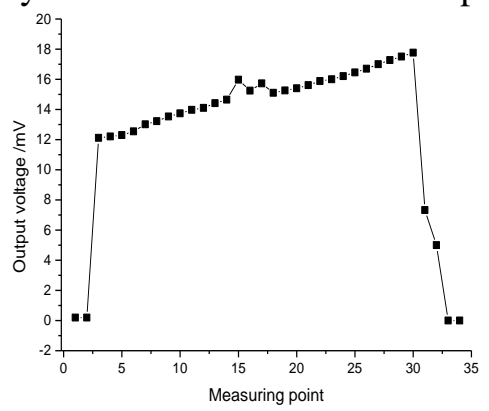

Figure 7. Measurement of Output Voltage

Figure8 shows the error of measurement frequency according to the equationError! Reference source not found.. From Fig.8, it is can be seen that the value of measurement error is big when the frequency below $3 \mathrm{~Hz}$ or high $30 \mathrm{~Hz}$. Furthermore, the error changes small along with the increasing of frequency and the average error between $3 \mathrm{~Hz}$ and $30 \mathrm{~Hz}$ is $1.78 \%$, which suggest the precision of this system is very good.

$$
e_{i}=\frac{f_{i}-M_{i}}{M_{i}} \times 100 \%
$$

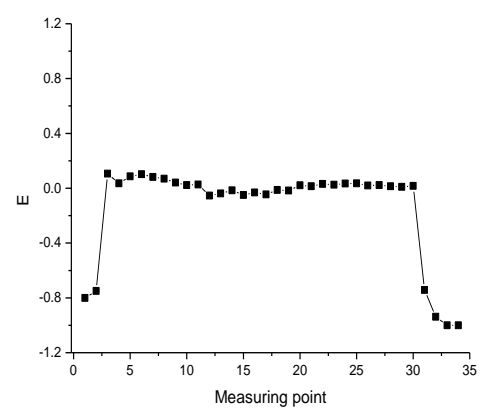

Figure 8. Measurement Error of Frequency

Besides the error, we import two evaluation indexes, the first is frequency linearity, which is calculated by equationError! Reference source not found., and the other is voltage sensitivity, which is calculated by equationError! Reference source not found.

$$
e_{L}= \pm \frac{\Delta L_{\max }}{y_{F S}} \times 100 \%
$$

Where, $\Delta L_{\max }$ is the max vertical value between output frequency and real frequency in Figure6, the valid frequency range is from $3 \mathrm{~Hz}$ to $30 \mathrm{~Hz}$ definitely, $y_{F S}$ is the theoretical max measurement range, and the value of $y_{F S}$ is $30 \mathrm{~Hz}$. It is can be found that $\Delta L_{\max }$ is $0.7 \mathrm{~Hz}$, so the frequency linearity $e_{L}$ is $2.25 \%$. By the same method, we could get the voltage linearity is $2.64 \%$.

$$
K=\frac{\Delta y}{\Delta x}
$$

Sensitivity is the ratio between output increment and input increment, which is the slope when the situation is linear system. So the voltage sensitivity of this system could be got as:

$$
K=\frac{17.77-12.12}{30-3}=0.135 m v / H z
$$




\section{Conclusion}

The experiment results show that the developed infrasonic sound sensor based on PVDF in this paper has good performance and high precision, furthermore it has advantage of light structure, strong anti-jamming capability, stability, which makes it could meet the design requirements, and has a certain practical value. Following are main conclusions of this paper:

(1) The frequency range of designed infrasonic sound sensor is from $3 \mathrm{~Hz}$ to $30 \mathrm{~Hz}$.

(2) When a given frequency values between $3 \mathrm{~Hz}$ to $30 \mathrm{~Hz}$, the voltage steady increase slowly, illustrate the sensor works stability and can meet the design requirement.

(3) The frequency error is $1.78 \%$ and frequency linearity is $2.25 \%$.

(4) The voltage linearity is $2.64 \%$, and voltage Sensitivity is $0.135 \mathrm{mv} / \mathrm{Hz}$.

\section{References}

[1] P. Audrain, P. Masson and A. Berry , "The Use of PVDF Strain Sensing in Active Control of Structural Intensity in Beams", Journal of intelligent material systems and structures, vol. 15, no. 5, (2004), pp. 319-327.

[2] C J. Sherry, M C. Cook and J R. Jauchem, "The effects of infrasound on rhesus monkey performance of a continuous compensatory tracking task [J]", Journal of Low Frequency Noise, Vibration and Active Control, vol. 27, no. 1, (2008), pp. 53-64.

[3] C. Chi, Z. Huijun and W. Xiaobin, "The Leaked Detection System of Gas Pipeline which based on Infrasonic Wave", Reston, VA, USA: American Society of Civil Engineers, (2013).

[4] J B. Johnson, J F. Anderson and R E. Anthony, "Detecting geyser activity with infrasound", Journal of Volcanology and Geothermal Research, vol. 256, (2013), pp. 105-117.

[5] J. Caplan-Auerbach, A. Bellesiles and J K. Fernandes, "Estimates of eruption velocity and plume height from infrasonic recordings of the 2006 eruption of Augustine Volcano", Alaska, Journal of Volcanology and Geothermal Research, vol. 189, no. 1-2, (2010), pp. 12-18.

[6] A. Kogelnig, J. Hubl and E. Surinach, "A study of infrasonic signals of debris flows", Padua, Italy: American Society of Civil Engineers (ASCE), (2011).

[7] S. Nomura, M. Hasegawa-Ohira and Y. Hanasaka, "Human pulse wave measurement by MEMS-ECM sensor", Rome, Italy: IADIS, (2011).

[8] J. Chilo and T. Lindblad, "Hardware implementation of 1D wavelet transform on an FPGA for infrasound signal classification", EEE Transactions on Nuclear Science, vol. 55, no. 1, (2008), pp. 9-13.

[9] F M. Ham, K. Rekab and R. Acharyya, "Infrasound signal classification using parallel RBF neural networks", International Journal of Signal and Imaging Systems Engineering, vol. 1, no. 3-4, (2008), pp. 155-167.

[10] X. Xue, Y. Xia and X. Cui, "Analysis of exceptional infrasound signal before Earthquakes with MATLAB”, Beijing Gongye Daxue Xuebao / Journal of Beijing University of Technology, vol. 34, (2008), pp. 117-122.

[11] S. Chai, L. Li and X. Gang, "Measurement and analysis of infrasound wave in the process of low carbon steel damage", Key Engineering Materials, vol. 439-440, (2010), pp. 698-703.

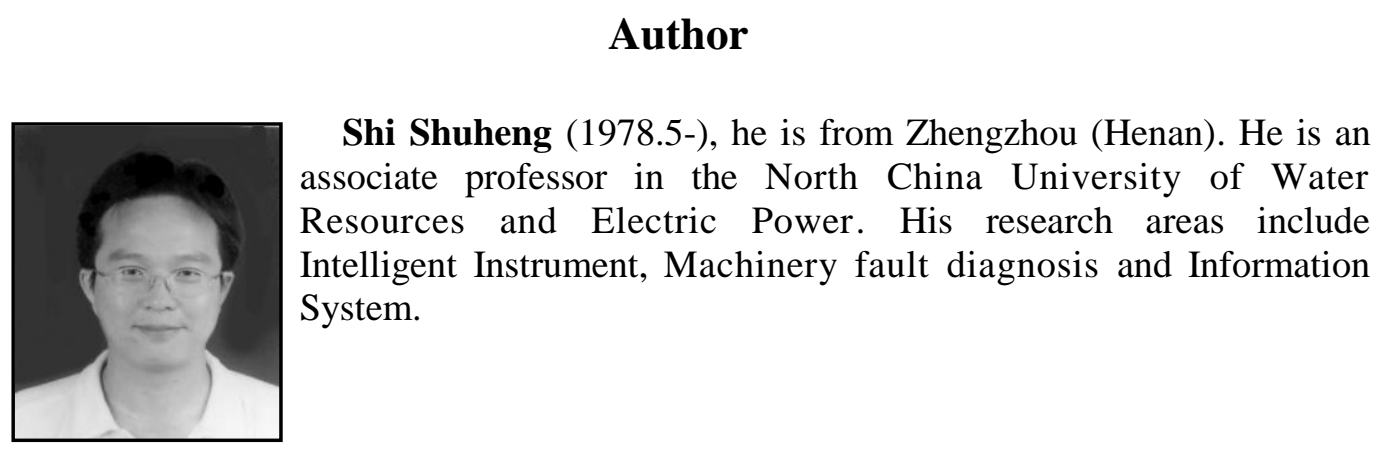

